Proc. Computational Structural Engineering, Yuan Y, Cui JZ and Mang H(eds.), Jun. 22-24, 2009, Springer, Shanghai: 127-135.

\title{
Simulation of Structural Collapse with Coupled Finite Element-Discrete Element Method
}

\author{
Xinzheng $\mathrm{Lu}^{1}$, Xuchuan $\operatorname{Lin}^{1}$ and Lieping $\mathrm{Ye}^{1}$ \\ ${ }^{1}$ Department of Civil Engineering, Tsinghua University, Beijing 100084, P.R. China
}

\begin{abstract}
Structural progressive collapse is a great threat to life safety and therefore it is necessary to study its mechanism in detail. Numerical simulation is significant to study the whole process of progressive collapse in structural level. Since collapse is a complicated procedure from continuum into discrete fragments, numerical model should be competent in nonlinear deformation before collapse and breaking and crashing of fragments after collapse. Coupled Finite elementdiscrete element method on simulating structural progressive collapse is proposed to meet the requirements. Relatively accurate models, such as fiber model and multi-shell shell model, are introduced to construct the finite element model of structure. In the analysis, the failed finite elements will be removed and replaced with granular discrete elements according to the criteria of equivalent total mass and volume so that the impacting and heaping of fragments can be taken into account. The sample with the coupled method shows that this method not only possesses the advantages of finite element method but also simulates the behavior of fragments well.
\end{abstract}

Keywords: Finite element, discrete element, coupling calculation, progressive collapse, numerical model.

\section{Introduction}

Progressive collapse causes great casualties and property losses, so progressive collapse should be strictly avoided in structural design .Though buildings which meet the present design codes generally possess enough collapse resistant capacity under conventional loads, however, it is important to carry out further detailed research on structural progressive collapse. Since collapse is an ultimate safety state of a structure, with a better understanding of collapse state, a better evaluation of a building's safety margin can be obtained. It is the typical mode of progressive collapse that failure first occurs at weak part of a structure and if there is no sufficient alternative loading path, then failure expands to the whole 
structure. Therefore, the study on collapse helps to find and enhance the weak part of a structure and can effectively improve its collapse resistant performance.

Since progressive collapse is a structural behaviour and buildings are generally huge, large scale tests are often too difficult to carry out with the limitation of time and cost. Therefore, numerical simulation is a significant method to study progressive collapse. Besides accidental impact and blast, rare strong earthquakes like Wenchuan Earthquake, which was more than nine degree in seismic intensity in a region with seven-degree design intensity, can unavoidably cause massive local failures. How to control the expansion of local failure to ensure the overall stability of structure is an important aspect of structural safety research. To investigate this problem, collapse process especially the expanding process of local damage has to be studied and simulation on the whole process of progressive collapse is necessary.

Collapse is a complex process from continuous body into discrete one, which puts forward high requirements for its numerical model. The model should not only can simulate behaviours before collapse, such as nonlinear deformation and energy dissipation, but also can simulate the rigid-body displacement of fragments and impact among damaged components after collapse begins. Many numerical methods were proposed to simulate this two-stage process and the existing numerical methods can be roughly classified into two categories, finite element method (FEM) and non-continuum mechanics method. Some scholars simulate the collapse process with dynamic finite element software LS-DYNA. Lu \& Jiang (2001) simulated the collapse of World Trade Centre and the numerical results are close to the real conditions. Liu et al (2007) and Shi et al (2007) constructed 3-D solid-element models to simulate progressive collapse due to impact of blast load. Lu et al(2007) and Miao et al (2007) developed fibber model THUFIBER based on commercial finite element software MSC.MARC and the model gave a good simulation on the collapse mechanism of RC frames under different loads. K. Khandelwal \& Tawil (2005) introduced multi-scale finite element models into the simulation of progressive collapse of an eight-story steel frames under blast loads. In the multi-scale method, micro scale models are validated by experimental results, macro scale models by micro scale models and structural scale models by macro scale models????. This method allows researchers to accurately and economically study the potential for progressive collapse in steel building frames. Isobe and Tsuda (2003) applied a new finite element code using Adaptively Shifted Integration (ASI) technique with a linear Timoshenko beam element to the seismic collapse analysis of RC framed structures. In the beam element, the fracture of a section was simulated by shifting the numerical integration point with simultaneous release of the resultant forces. As to non-continuum mechanics method, Qin et al (2001) and Sun et al (2002) proposed a particle-truss model for collapse analysis of the RC bridge and the reinforcement concrete is modelled as particles connected with nonlinear springs. The model had a good simulation of failure behaviour after collapse. Xuan et al (2003) and Wang \&Lü (2004) put forward a discrete element model for collapse simulation of RC frame with shear- 
type deformation. In this model, every floor is a rigid body without rotation displacement and the adjacent stories are connected with an axial spring and a shear spring. Zhao (2008) proposed 2-D DEM model for collapse of RC frame and the frame was discretized into rectangular elements. Miao et al (2005) employed discrete element method (DEM) to simulate the collapse process of a threedimension masonry structures under earthquakes. The model was made up of block elements connected with springs in both transverse and longitudinal directions and the numerical results are close to those of the shaking table test. Cui et al (2002) employed 3-D rigid body-spring element method to simulate the collapse process of Malpasset Arch Dam.

Finite element method (FEM) appear good performance in simulating structural behaviour before collapse, but has difficulties in dealing with the behaviours in the process of progressive collapse, such as fracture of components, impact and stack load of fragments. Yet non-continuum mechanics method is much more competent in simulates the behaviour after collapse. If these two kinds of methods can work together and take their own advantages, the simulation will be largely improved. According to this point, Munjiza and Bangash (2004) proposed a combined finite-discrete element model and it produced good numerical results for structures made up of RC column or column type members, though it failed to take account of slabs and non-structural components. In the paper, coupled finite element-discrete element method for progressive collapse of RC buildings was proposed and its technique details are given. The simulation results show that this new method not only keeps the advantages of FEM but also gives a good simulation on impact and stacking of fragments.

\section{Finite element models for collapse}

Structural components of RC buildings mainly consist of beam, column, wall and floor slab. To obtain a more accurate result, fiber beam element model (Wang et al 2007) is proposed for beam and column, and multi-layer shell element model (Men et al 2006) for wall and slab, detailed illustration can be found in $\mathrm{Lu}$ et al 2008.

\section{Discrete element model for collapse}

Discrete element method (DEM) is used to study assemblies of distinct interacting particles or general shaped bodies. One of the common models is constructed with spherical particles which are particularly called as granular discrete element. Granular discrete element method (GDEM) not only possesses the basic motion features of non-continuum, but also has a simple contact algorithm. The coupled 
4 Simulation of Structural Progressive Collapse with Coupled Finite Element-Discrete Element Method

finite element-discrete element method uses granular discrete elements to simulate the fragments in the collapse of structures. On one hand, the size of finite element is very small compared to the structural members and the discrete elements are generated from the further discretization of finite elements, so the effect of the shape of discrete elements on the collapse process is quite small. On the other hand, the main concern on this collapse model is the effects of impact and stack load in the collapse process.

\subsection{Generation of granular discrete element}

Once a four-node quad finite element is deactivated (removed from the model), nine granular discrete elements will be generated instead of this quad element, shown as figure 1 . The generation process keeps the equivalent total mass and volume and the initial motion states of every discrete element, such as coordinates, velocities and accelerations are obtained with linear interpolation based on the four nodes of the deactivated finite element.

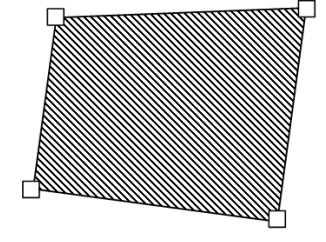

“Deactivated" finite element

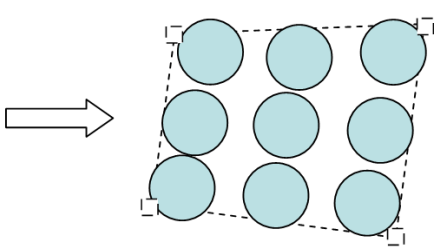

9 discrete elements

Figure 1 The “deactivated" element is replaced with 9 discrete elements

\subsection{Motion law of granular discrete element}

The sphere element can be regarded as a particle when moving, the motion equation at the moment $t_{i}$ is

$$
m \ddot{u}\left(t_{i}\right)+\eta \dot{u}\left(t_{i}\right)=p\left(t_{i}\right)
$$

where $\boldsymbol{m}$ is mass of the discrete element, $\mathbf{p}$ is vector of external forces at $t_{i}, \mathbf{u}$ is displacement vector at $t_{i}, \eta$ is damping coefficient.

Central difference method is applied to compute the motion state. If the time step is constant, that is $\Delta t_{i}=\Delta t$, we get

$$
\begin{aligned}
& \dot{u}_{i}=\left(u_{i+1}-u_{i-1}\right) /(2 \Delta t) \\
& \ddot{u}_{i}=\left(u_{i+1}-2 u_{i}+u_{i-1}\right) / \Delta t^{2}
\end{aligned}
$$

By substitution of Eq. (2) and Eq. (3) into Eq. (1), we obtain 


$$
\left(\frac{m}{\Delta t^{2}}+\frac{c}{2 \Delta t}\right) u_{i+1}=p\left(t_{i}\right)+\frac{2 m}{\Delta t^{2}} u_{i}-\left(\frac{m}{\Delta t^{2}}-\frac{c}{2 \Delta t}\right) u_{i-1}
$$

Then displacement, velocity and acceleration of a discrete element at every moment can be calculated through Eq.(2), Eq.(3) and Eq.(4).

\subsection{Contact judgment}

Contact between two discrete elements happens if the distance between their spherical centers is no larger than the total length of their radiuses. The contact criterion (Wei et al 2008) for element $i$ and element $j$ is

$$
\begin{aligned}
& \left(r_{i}+r_{j}\right) \times C N C \geq R_{i j} \\
& R_{i j}=\sqrt{\left(x_{i}-x_{j}\right)^{2}+\left(y_{i}-y_{j}\right)^{2}+\left(z_{i}-z_{j}\right)^{2}}
\end{aligned}
$$

where $\mathrm{r}_{\mathrm{i}}, \mathrm{r}_{\mathrm{j}}$ are radiuses of element $\boldsymbol{i}$ and element $\boldsymbol{j}$ respectively, $\mathrm{R}_{\mathrm{ij}}$ is the distance between the spherical centers of element $i$ and element $\boldsymbol{j}$, CNC is a coefficient relevant to the medium where elements soak, usually $\mathrm{CNC} \geq 1.0$ and $\mathrm{CNC}=1.0$ in this paper.

To simulate the impact of fragments to structures, contact between discrete element and finite element needs to be accomplished. The finite element is simplified as 9 spheres with the similar process in chapter 3.1 and thus, the contact between discrete element and finite element is transformed into that between two discrete elements.

\section{Program implementation and example}

Coupled finite element-discrete element method was implemented through secondary development of commercial finite element software MSC.MARC 2005 and the details are as follows:

(a) Deactivation of finite elements and generation of discrete elements.

When a finite element is damaged, it will be deactivated (removed) by a user subroutine called UACTIVE. This process is controlled by the deactivating criteria ( $\mathrm{Lu}$ et al 2008). Once the deformation of a finite element is too large, the element will be removed and the critical deformation can be determined in terms of maximum strain. The criteria defined in present work is the same as those in $\mathrm{Lu}$ et al 2008 and other criteria can also be defined by UACTIVE.

The deactivated element is transformed into nine discrete elements as mentioned in chapter 3.1. The program gives the initial condition of discrete elements and record the motion state, mass and volume in the whole process. 
6 Simulation of Structural Progressive Collapse with Coupled Finite Element-Discrete Element Method

(b) Contact.

During collapse, impact and stack load of fragments have a significant effect on the stories below, so contact should be taken into account. There are three types of contact, contact between finite elements, contact between discrete elements and contact between finite elements and discrete elements. The first type of contact can be directly set in finite element software MSC.MARC and the second and third can be defined by user subroutine UBGITR. UBGITR will be called at the beginning of each iteration.

(c) Effect of impact and stack loads.

When the discrete elements collide with finite element structure, contact happens and user subroutine FORCDT will exert contact forces to the nodes of relevant finite elements.

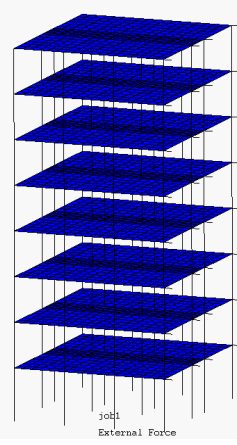

(a) $\mathrm{t}=0.0 \mathrm{~s}$

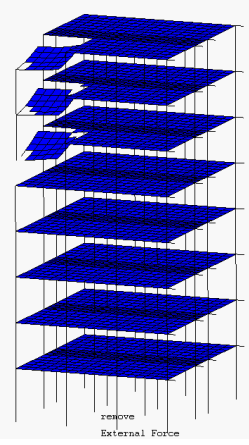

(b) $\mathrm{t}=1.0 \mathrm{~s}$

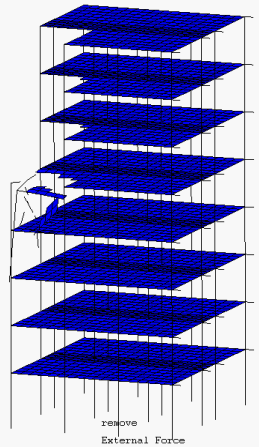

(c) $\mathrm{t}=2.0 \mathrm{~s}$

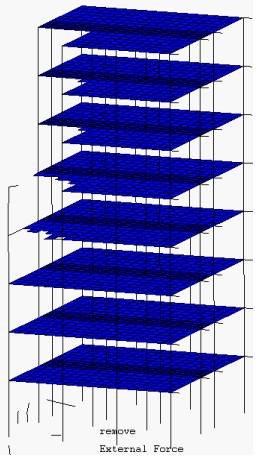

(d) $\mathrm{t}=3.0 \mathrm{~s}$

Figure 2 Collapse procedure simulated with finite element method (FEM)

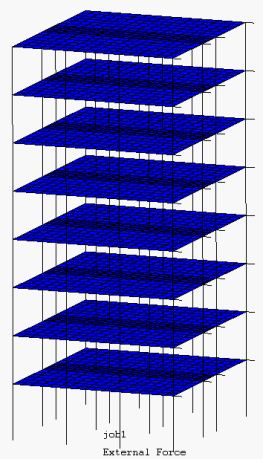

(a) $\mathrm{t}=0.0 \mathrm{~s}$

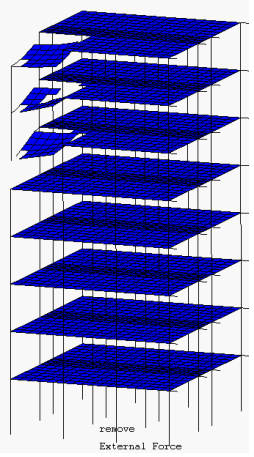

(b) $\mathrm{t}=1.0 \mathrm{~s}$

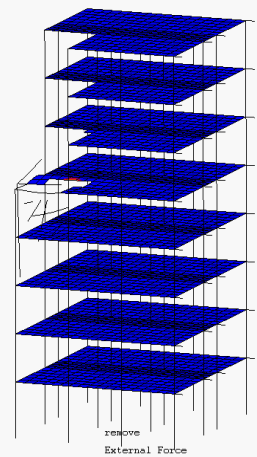

(c) $\mathrm{t}=2.0 \mathrm{~s}$

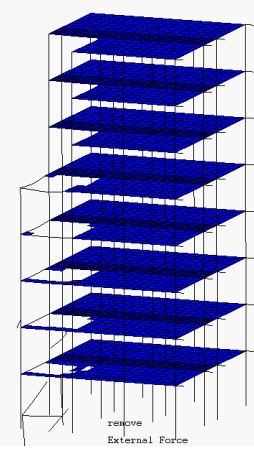

(d) $\mathrm{t}=3.0 \mathrm{~s}$

Figure 3 Collapse procedure simulated with coupled Finite element-discrete element method 
A numerical example of an eight-story frame is carried out to observe the effect of coupled finite element-discrete element method. Columns and beams are modeled with fiber beam element model and slabs with multi-layer shell element model. Progressive collapse occurred after the corner column of the fifth story was removed. Two methods were applied to simulate collapse, one is FEM and the other is coupled finite element-discrete element method. The results from FEM are listed in figure 2 and those from coupled finite element-discrete element method (F/DEM) are listed in figure 3. It is noted that discrete elements can not be seen in Fig.11 because the program hasn' $t$ support this function. At the beginning of collapse (before $t=2.0 \mathrm{~s}$ ), structural response of FEM is similar to that of F/DEM. When $\mathrm{t}=1.0 \mathrm{~s}$, the slabs above the removed column break from the slabs around and drop off. When $\mathrm{t}=2.0 \mathrm{~s}$, the fallen slabs crash through the fifth floor. However, these two methods appear distinct results at 3.0s. With FEM, the progressive collapse is stopped after the fourth floor slabs drop. With F/DEM, all the floor slabs below the removed column are smashed. It is shown that the model based on F/DEM shows a better simulation on impact and stack load in the process of progressive collapse than that based on FEM.

\section{Conclusions}

Numerical simulation is an important tool to study progressive collapse. Collapse of structure is a complicated process from continuum into discrete bodies, which put forward requirements for the numerical model. The model should not only simulate the nonlinear behaviors before collapse, but also simulate impact and stack load of fragments during the collapse. Finite element method has advantages in simulating continuum while discrete element method in simulating the noncontinuous behaviors after collapse starts. Coupled finite element-discrete element method (F/DEM) is proposed and implemented based on existing finite element software. Keeping the advantages of FEM, the method applies DEM to simulate the fragments in collapse. The numerical results show that this coupled FEMDEM method has a better simulation on impact and stack load in progressive collapse than FEM.

\section{Acknowledgements}

This research is supported by National Science Foundation of China (No. $50808106 \& 90815025)$ 
8 Simulation of Structural Progressive Collapse with Coupled Finite Element-Discrete Element Method

\section{References}

Xin-zheng Lu, Jian-jing Jiang (2001). Dynamic finite element simulation for the collapse of world trade center. China Civil Engineering Journal,34(6), 8-11 [In Chinese].

Dong Qin, Li-chu Fan(2001). Numerical simulation on collapse process of reinforced concrete structures. Journal of Tongji University, 29(1), 80-83 [in Chinese].

Yu-zhu Cui, Chu-han Zhang,et al (2002). Numerical modeling of dam-foundation failure and simulation of arch dam collapse. Shuili Xuebao, 6,1-8 [in Chinese].

Li-ming Sun, Dong Qin, Li-chu Fan (2002). A new model for collapse analysis of reinforced concrete. China Civil Engineering Journal, 35(6), 53-58 [in Chinese].

D. Isobe, M. Tsuda(2003). Seismic collapse analysis of reinforced concrete framed structures using the finite element method. Earthquake Engineering and Structural Dynamics, 32, 2027-2046.

Gang Xuan, Xiang-lin Gu, Xi-lin Lü (2003). Numerical analysis of collapse process for RC frame structures subjected to strong earthquakes. Earthquake Engineering and Engineering Vibration, 23(6), 24-30 [in Chinese].

A. Munjiza, T. Bangash, N.W.M. John(2004). The combined finite-discrete element method for structural failure and collapse. Engineering Fracture Mechanics, 469-483.

Qiang Wang, Xi-lin Lü(2004). Application of the DEM to the seismic response analysis of frame structures. Earthquake Engineering and Engineering Vibration, 24(5), 73-78 [in Chinese].

K. Khandelwal, and S. El-Tawil(2005). Multiscale computational simulation of progressive collapse of steel frames. Proceedings of the ASCE Structures Congress, May 2005.

$\mathrm{Ji}$-jun Miao, Xiang-lin Gu, et al (2005). Numerical simulation analysis for the collapse response of masonry structures under earthquakes. China Civil Engineering Journal, 38(9), 45-52 [in Chinese].

Jun Men, Xin-zheng Lu, et al (2006). Application of multi-layer model in shell wall computation. Protective Structure 28:3,9 13 [in Chinese].

Jing-bo Liu, Yang-bing Liu, Jian-guo Yang (2007). Simulation of earthquake ruins structure for the Earthquake Rescue Training Base of China. Journal of Disaster Prevention and Mitigation Engineering, 27(suppl.), 428-432 [In Chinese].

Yan-chao Shi, Zhong-xian Li, Hong He(2007). Numerical analysis of progressive collapse of reinforced concrete under blast loading. Journal of PLA University of Science and Technology, 18(6), 652-658 [in Chinese].

Xin-zheng Lu, Yan-sheng Zhang, Jian-jing Jiang (2007). Simulation for the collapse of reinforced concrete frame by blasting based on fiber model. Blasting, 24(2), 1-6[in Chinese].

Xun-liu Wang, Xin-zheng Lu, Lie-ping Ye(2007). Numerical Simulation for the Hysteresis Behavior of RC Columns under Cyclic Loads. Engineering Mechanics, 24(12),76-81 [in Chinese] [in Chinese].

Zhi-wei Miao, Xin-zheng Lu, et al.(2007). Simulation for the collapse of RC frame tall buildings under earthquake disaster. Computational Mechanics, Proceedings of ISCM 2007, July 30 August 1, Beijing,China.

Feng-lei Zhao (2008). Simulation analysis for collapse of reinforced concrete frame structures under earthquake based on the discrete element method. Master thesis of Tongji University, Shanghai [in Chinese].

Long-hai Wei, Chun-guang Chen, et al(2008). Study on three-dimensional discrete element and parameter adoption. Journal of Chongqing Jiaotong University (Natural Science), 27(4), 618$621,629$.

Xin-zheng Lu, Xu-chuan Lin,et al (2008). Numerical Simulation for the progressive collapse of concrete building due to earthquake. The 14th World Conference on Earthquake Engineering, Qctober 12-17, 2008, Beijing, China. 